\title{
MINIMUM ENERGY CONTROL OF POSITIVE TIME-VARYING LINEAR SYSTEMS
}

\author{
Tadeusz KACZOREK ${ }^{\star}$
}

*Faculty of Electrical Engineering, Bialystok University of Technology, Wiejska 45D, 15-351 Bialystok, Poland

kaczorek@isep.pw.edu.pl

received 22 May 2015, revised 11 December 2015, accepted 14 December 2015

\begin{abstract}
The minimum energy control problem for the positive time-varying linear systems is formulated and solved. Sufficient conditions for the existence of solution to the problem are established. A procedure for solving of the problem is proposed and illustrated by a numerical example.
\end{abstract}

Keywords: Positive, Continuous-Time, Minimum Energy Control, Procedure

\section{INTRODUCTION}

A dynamical system is called positive if its trajectory starting from any nonnegative initial state remains forever in the positive orthant for all nonnegative inputs. An overview of state of the art in positive system theory is given in the monographs (Farina and Rinaldi,2000; Kaczorek, 2001b). Variety of models having positive behavior can be found in engineering, economics, social sciences, biology and medicine, etc.

The positive fractional linear systems have been investigated in Kaczorek (2008a, 2011c,d, 2012). Stability of fractional linear 1D discrete-time and continuous-time systems has been investigated in the papers (Busłowicz, 2008; Dzieliński and Sierociuk, 2008; Kaczorek, 2012) and of 2D fractional positive linear systems in Kaczorek (2009). The notion of practical stability of positive fractional discrete-time linear systems has been introduced in Kaczorek (2008b). The minimum energy control problem for standard linear systems has been formulated and solved by Klamka (1976, 1977, 1983, 1991, 1993, 2010) and for 2D linear systems with variable coefficients in Kaczorek and Klamka (1986). The controllability and minimum energy control problem of fractional discrete-time linear systems has been investigated by Klamka (2010). The minimum energy control of fractional positive continuous-time linear systems has been addressed in Kaczorek (2014b) and for descriptor positive discrete-time linear systems in Kaczorek (2014a).

In this paper the minimum energy control problem for positive time-varying linear systems will be formulated and solved.

The paper is organized as follows. In section 2 the basic definitions and theorems of the positive time-varying linear systems are recalled and the necessary and sufficient conditions for the reachability of the positive systems are given. The minimum energy control problem of the positive time-varying linear systems is formulated and solved in section 3. Sufficient conditions for the existence of solution of the problem are established and a procedure for computation of the optimal inputs and the minimum value of the performance index are also presented. Concluding remarks are given in section 4 .

The following notation will be used: $\mathfrak{R}-$ the set of real num- bers, $\mathfrak{R}^{n \times m}$ - the set of $\mathrm{n} \times \mathrm{m}$ real matrices, $\mathfrak{R}_{+}^{n \times m}-$ the set of $n \times m$ matrices with nonnegative entries and $\mathfrak{R}_{+}^{n}=\mathfrak{R}_{+}^{n \times 1}$, $M_{n}$ - the set of $n \times n$ Metzler matrices (real matrices with nonnegative off-diagonal entries), $I_{n}$ - the $n \times n$ identity matrix, $A^{T}$ - the transpose matrix $A$.

\section{POSITIVE TIME-VARYING LINEAR SYSTEMS AND THEIR REACHABILITY}

Consider the time-varying linear system

$\dot{x}(t)=A(t) x(t)+B(t) u(t)$

where $x(t) \in \mathfrak{R}^{n}$ and $u(t) \in \mathfrak{R}^{m}$ are the state and input vectors and $A(t) \in \mathfrak{R}^{n \times n}, B(t) \in \mathfrak{R}^{n \times m}$ with continuous-time entries.

The solution of equation (2.1) has the form

$$
\begin{aligned}
& x(t)=\Phi\left(t, t_{0}\right) x_{0} \\
& +\int_{0}^{t} \Phi(t, \tau) B(t) u(\tau) d \tau, \quad x\left(t_{0}\right)=x_{t_{0}}
\end{aligned}
$$

where $\Phi\left(t, t_{0}\right)$ is the fundamental matrix defined by

$$
\begin{aligned}
\Phi\left(t, t_{0}\right)=I_{n}+\int_{t_{0}}^{t} A(\tau) d \tau & t \\
& +\int_{t_{0}}^{t} A(\tau) \int_{t_{0}}^{\tau} A\left(\tau_{1}\right) d \tau_{1} d \tau+\ldots
\end{aligned}
$$

If $A\left(t_{1}\right) A\left(t_{2}\right)=A\left(t_{2}\right) A\left(t_{1}\right)$ for $t_{1}, t_{2} \in\left[t_{0}, \infty\right)$, then (2.3a) takes the form (Gantmacher, 1959)

$\bar{\Phi}\left(t, t_{0}\right)=\exp \left(\int_{t_{0}}^{t} A(\tau) d \tau\right)$.

The fundamental matrix $\Phi\left(t, t_{0}\right)$ satisfies the matrix differential equation

$\dot{\Phi}\left(t, t_{0}\right)=A(t) \Phi\left(t, t_{0}\right)$ 
and the initial condition $\Phi\left(t_{0}, t_{0}\right)=I_{n}$.

Lemma 2.1. The fundamental matrix satisfies

$\Phi\left(t, t_{0}\right) \in \Re_{+}^{n \times n}$ for $t \geq t_{0}$

if and only if the off-diagonal entries $a_{i j}, i \neq j, i, j=1,2, \ldots, n$ of the matrix $A(t)$ satisfy the condition

$\int_{t_{0}}^{t} a_{i j}(\tau) d \tau \geq 0$ for $i \neq j, i, j=1,2, \ldots, n$.

Proof is given in Kaczorek (2001a).

Definition 2.1. The system (2.1) is called the (internally) positive if and only if $x(t) \in \Re_{+}^{n}, t \geq t_{0}$ for any initial conditions $x_{t_{0}} \in$ $\mathfrak{R}_{+}^{n}$ and all inputs $u(t) \in \mathfrak{R}_{+}^{m}, t \geq t_{0}$.

Theorem 2.1. The time-varying linear system (2.1) is positive if and only if the off-diagonal entries of the matrix $A(t)$ satisfy the condition (2.6) and $B(t) \in \mathfrak{R}_{+}^{n \times m}$ for $t \geq t_{0}$.

Definition 2.2. The system (2.1) is called reachable in time $t_{f}-t_{0}$ if for any given final state $x_{f} \in \mathfrak{R}_{+}^{n}$ there exists an input $u(t) \in \mathfrak{R}_{+}^{m}$, for $t \in\left[t_{0}, t_{f}\right]$ that steers the state of the system from zero initial state $x\left(t_{0}\right)=x_{t_{0}}$ to the state $x_{f}$, i.e. $x\left(t_{f}\right)=$ $x_{f}$.

A real square matrix is called monomial if each its row and each its column contains only one positive entry and the remaining entries are zero.

Theorem 2.2. The positive system (2.1) is reachable in time $t_{f}-t_{0}$ if and only if

$R_{f}=\int_{t_{0}}^{t_{f}} \Phi\left(t_{f}, \tau\right) B(\tau) B^{T}(\tau) \Phi^{T}\left(t_{f}, \tau\right) d \tau$

is a monomial matrix. The input vector which steers the state of the system (2.1) from $x\left(t_{0}\right)=x_{t_{0}}$ to $x_{f}$ is given by

$u(t)=B^{T}(\tau) \Phi^{T}\left(t_{f}, t\right) R_{f}^{-1} x_{f}$ for $t \in\left[t_{0}, t_{f}\right]$.

Proof. It is well-known (Kaczorek, 2001b) that the inverse matrix $R_{f}^{-1} \in \mathfrak{R}_{+}^{n \times n}$ if and only if the matrix $R_{f}$ is monomial. In this case the input $u(t) \in \mathfrak{R}_{+}^{m}$ given by (2.8) steers the state of the system from $x\left(t_{0}\right)=x_{t_{0}}$ to the state $x_{f}$. Substituting (2.8) into (2.2) for $t=t_{f}$ and $x\left(t_{0}\right)=x_{t_{0}}$ we obtain

$$
\begin{aligned}
& x\left(t_{f}\right)=\int_{t_{0}}^{t_{f}} \Phi\left(t_{f}, \tau\right) B(\tau) B^{T}(\tau) \Phi^{T}\left(t_{f}, \tau\right) R_{f}^{-1} x_{f} d \tau \\
& \quad=\left[\int_{t_{0}}^{t_{f}} \Phi\left(t_{f}, \tau\right) B(\tau) B^{T}(\tau) \Phi^{T}\left(t_{f}, \tau\right) d \tau\right] R_{f}^{-1} x_{f}=x_{f} .
\end{aligned}
$$

Therefore, the positive system (2.1) is reachable in time $t_{f}-t_{0}$ if and only if the matrix (2.7) is monomial. $\square$

\section{MINIMUM ENERGY CONTROL PROBLEM}

Consider the positive system (2.1) reachable in time $t_{f}-t_{0}$. If the system is reachable in time $t \in\left[t_{0}, t_{f}\right]$, then usually there exists many different inputs $\mathrm{u}(\mathrm{t}) \in \mathfrak{R}_{+}^{\mathrm{n}}$ that steers the state of the system from $x\left(t_{0}\right)=x_{t_{0}}=0$ to $x_{f}=x\left(t_{f}\right) \in \mathfrak{R}_{+}^{n}$. Among these inputs we are looking for an input $u(t) \in \mathfrak{R}_{+}^{n}$ that minimizes the performance index

$I(u)=\int_{t_{0}}^{t_{f}} u^{T}(\tau) Q u(\tau) d \tau$

where $Q \in \Re_{+}^{n \times n}$ is a symmetric positive defined matrix and $Q^{-1} \in \mathfrak{R}_{+}^{n \times n}$.

The minimum energy control problem for the positive time- varying linear systems (2.1) can be stated as follows: Given the matrices $A(t), B(t)$ and $Q \in \Re_{+}^{n \times n}$ of the performance index (3.1), $x_{f} \in \Re_{+}^{n}, t_{0}$, and $t_{f}>0$, find an input $u(t) \in \mathfrak{R}_{+}^{n}$ for $t \in\left[t_{0}, t_{f}\right]$ that steers the state vector of the system from $x_{t_{0}}=0$ to $x_{f} \in \mathfrak{R}_{+}^{n}$ and minimizes the performance index (3.1).

To solve the problem we define the matrix

$$
\begin{aligned}
& W=W\left(t_{f}, Q\right) \\
& =\int_{t_{0}} \Phi\left(t_{f}, \tau\right) B(\tau) Q^{-1} B^{T}(\tau) \Phi^{T}\left(t_{f}, \tau\right) d \tau
\end{aligned}
$$

From (3.2) and Theorem 2.2 it follows that the matrix (3.2) is monomial if and only if the fractional positive system (2.1) is reachable in time $\left[t_{0}, t_{f}\right]$. In this case we may define the input

$\hat{u}(t)=Q^{-1} B^{T}(t) \Phi^{T}\left(t_{f}, t\right) W^{-1} x_{f}$ for $t \in\left[t_{0}, t_{f}\right]$.

Note that the input (3.3) satisfies the condition $u(t) \in \mathfrak{R}_{+}^{\mathrm{n}}$ for $t \in\left[t_{0}, t_{f}\right]$ if

$Q^{-1} \in \mathfrak{R}_{+}^{n \times n}$ and $W^{-1} x_{f} \in \Re_{+}^{n}$.

Theorem 3.1. Let the positive system (2.1) be reachable in time $\left[t_{0}, t_{f}\right]$ and let $\bar{u}(t) \in \mathfrak{R}_{+}^{n}$ for $t \in\left[t_{0}, t_{f}\right]$ be an input that steers the state of the positive system (2.1) from $x_{t_{0}}=0$ to $x_{f} \in \mathfrak{R}_{+}^{n}$. Then the input (3.3) also steers the state of the system from $x_{t_{0}}=0$ to $x_{f} \in \Re_{+}^{n}$ and minimizes the performance index (3.1), i.e. $I(\hat{u}) \leq I(\bar{u})$.

The minimal value of the performance index (3.1) is equal to

$I(\hat{u})=x_{f}^{T} W^{-1} x_{f}$.

Proof. If the conditions (3.4) are met then the input (3.3) is well defined and $\hat{u}(t) \in \mathfrak{R}_{+}^{n}$ for $t \in\left[t_{0}, t_{f}\right]$. We shall show that the input steers the state of the system from $x_{t_{0}}=0$ to $x_{f} \in \Re_{+}^{n}$. Substitution of (3.3) into (2.2) for $t=t_{f}$ and $x_{t_{0}}=0$ yields

$$
\begin{aligned}
x & \left(t_{f}\right)=\int_{t_{0}}^{t_{f}} \Phi\left(t_{f}, \tau\right) B(\tau) \hat{u}(\tau) d \tau \\
& =\int_{t_{0}}^{t_{f}} \Phi\left(t_{f}, \tau\right) B(\tau) Q^{-1} B^{T}(\tau) \Phi^{T}\left(t_{f}, \tau\right) d \tau W^{-1} x_{f} \\
& =x_{f}
\end{aligned}
$$

since (3.2) holds. By assumption the inputs $\bar{u}(t)$ and $\hat{u}(t)$, $t \in\left[t_{0}, t_{f}\right]$ steers the state of the system from $x_{t_{0}}=0$ to $x_{f} \in \mathfrak{R}_{+}^{n}$. Hence

$$
\begin{aligned}
x_{f}=\int_{t_{0}}^{t_{f}} \Phi\left(t_{f}, \tau\right) B(\tau) \bar{u}(\tau) d \tau \\
=\int_{t_{0}}^{t_{f}} \Phi\left(t_{f}, \tau\right) B(\tau) \hat{u}(\tau) d \tau
\end{aligned}
$$

or

$\int_{t_{0}}^{t_{f}} \Phi\left(t_{f}, \tau\right) B(\tau)[\bar{u}(\tau)-\hat{u}(\tau)] d \tau=0$.

By transposition of (3.7b) and postmultiplication by $\mathrm{W}^{-1} \mathrm{x}_{\mathrm{f}}$ we obtain

$\int_{t_{0}}^{t_{f}}[\bar{u}(\tau)-\widehat{u}(\tau)]^{T} B^{T}(\tau) \Phi^{T}\left(t_{f}, \tau\right) d \tau W^{-1} x_{f}=0$.

Substitution of (3.3) into (3.8) yields 


$$
\begin{aligned}
& \int_{t_{0}}^{t_{f}}[\bar{u}(\tau)-\hat{u}(\tau)]^{T} B^{T}(\tau) \Phi^{T}\left(t_{f}, \tau\right) d \tau W^{-1} x_{f} \\
& \quad=\int_{t_{0}}^{t_{f}}[\bar{u}(\tau)-\hat{u}(\tau)]^{T} Q \hat{u}(\tau) d \tau=0 .
\end{aligned}
$$

Using (3.9) it is easy to verify that

$$
\begin{aligned}
& \int_{t_{0}}^{t_{f}} \bar{u}(\tau)^{T} Q \bar{u}(\tau) d \tau=\int_{t_{0}}^{t_{f}} \hat{u}(\tau)^{T} Q \hat{u}(\tau) d \tau \\
& \quad+\int_{t_{0}}^{t_{f}}[\bar{u}(\tau)-\hat{u}(\tau)]^{T} Q[\bar{u}(\tau)-\hat{u}(\tau)] d \tau .
\end{aligned}
$$

From (3.10) it follows that $\mathrm{I}(\hat{\mathrm{u}})<\mathrm{I}(\overline{\mathrm{u}})$ since the second term in the right-hand side of the inequality is nonnegative.

To find the minimal value of the performance index (3.1) we substitute (3.3) into (3.1) and we obtain

$$
\begin{aligned}
& I(\hat{u})=\int_{0}^{t_{f}} \hat{u}^{T}(\tau) Q \hat{u}(\tau) d \tau \\
& \quad=x_{f}^{T} W^{-1} \int_{0}^{t_{f}} \Phi\left(t_{f}, \tau\right) B(\tau) Q^{-1} B^{T}(\tau) \Phi^{T}\left(t_{f}, \tau\right) d \tau W^{-1} x_{f} \\
& \quad=x_{f}^{T} W^{-1} x_{f}
\end{aligned}
$$

since (3.2) holds. $\square$

From the above considerations we have the following procedure for computation the optimal inputs that steers the state of the system from $x_{t_{0}}=0$ to $x_{f} \in \mathfrak{R}_{+}^{n}$ and minimize the performance index (3.1).

\section{Procedure 3.1}

Step 1. Knowing the matrix $A(t)$ compute $\Phi(t)$.

Step 2. Using (3.2) compute the matrix $W$.

Step 3. Using (3.3) compute the input $\hat{u}(t)$.

Step 4. Using (3.5) compute the minimal value of the performance index.

Example 3.1. Consider the positive system (2.1) for $t_{0}=0$ with matrices

$A(t)=\left[\begin{array}{ll}2 & 0 \\ 0 & t\end{array}\right], \quad B(t)=\left[\begin{array}{ll}0 & e^{t} \\ \sqrt{t} & 0\end{array}\right]$

and the performance index (3.1) with

$Q=\left[\begin{array}{ll}2 & 0 \\ 0 & 2\end{array}\right]$.

By Theorems 2.1 and 2.2 the system is positive and reachable in time $t_{f}-t_{0}$. Therefore, there exists an input $\mathrm{u}(\mathrm{t})$ that steers the state of the system from zero state to $x_{f}=\left[\begin{array}{ll}2 & 1\end{array}\right]^{T}$ in time $t_{f}-t_{0}$ for $t_{0}=0, t_{f}=1$.

Using the Procedure 3.1 we obtain the following:

Step 1. Using (2.3a) we obtain

$$
\begin{aligned}
& \bar{\Phi}(1, \tau)=\exp \left(\int_{\tau}^{1} A(\tau) d \tau\right)= \\
& {\left[\begin{array}{ll}
\exp (2(1-\tau)) & 0 \\
0 & \exp \left(0.5\left(1-\tau^{2}\right)\right)
\end{array}\right] .}
\end{aligned}
$$

Step 2. Using (3.2), (3.13) and (3.14) we obtain

$$
\begin{aligned}
W & =\int_{0}^{t_{f}} \bar{\Phi}(1, \tau) B(\tau) Q^{-1} B^{T}(\tau) \bar{\Phi}^{T}(1, \tau) d \tau \\
& =\left[\begin{array}{ll}
0.25 e^{2}\left(e^{2}-1\right) & 0 \\
0 & 0.25(e-1)
\end{array}\right] .
\end{aligned}
$$

Step 3. Using (3.3) and (3.15) we have

$\hat{u}(t)=Q^{-1} B^{T}(t) \bar{\Phi}^{T}(1, t) W^{-1} x_{f}=$

$\left[\begin{array}{l}\frac{2 \sqrt{t}}{e-1} \exp \left(0.5\left(1-\tau^{2}\right)\right) \\ \frac{4 \exp (-t)}{e^{2}-1}\end{array}\right]$.
Step 4. The minimal value of the performance index

$$
\begin{aligned}
& I(\hat{u})=x_{f}^{T} W^{-1} x_{f}=\left[\begin{array}{ll}
2 & 1
\end{array}\right] \\
& {\left[\begin{array}{ll}
0.25 e^{2}\left(e^{2}-1\right) & 0 \\
0 & 0.25(e-1)
\end{array}\right]^{-1}\left[\begin{array}{l}
2 \\
1
\end{array}\right]} \\
& \quad=\frac{16}{e^{2}\left(e^{-2}-1\right)}+\frac{4}{e-1} .
\end{aligned}
$$

\section{CONCLUDING REMARKS}

Necessary and sufficient conditions for the reachability of the positive time-varying linear systems have been established (Theorem 2.2). The minimum energy control problem for the positive time-varying linear systems has been formulated and solved. Sufficient conditions for the existence of a solution to the problem has been given (Theorem 3.1). A procedure for computation of optimal input and the minimal value of performance index has been proposed. The effectiveness of the procedure has been demonstrated on the numerical example. The presented method can be extended to positive discrete-time linear systems and to fractional positive time-varying linear systems with bounded inputs.

\section{REFERENCES}

1. Busłowicz M. (2008), Stability of linear continuous time fractional order systems with delays of the retarded type, Bull. Pol. Acad. Sci. Tech., Vol. 56(4), 319-324.

2. Dzieliński A., Sierociuk D. (2008), Stability of discrete fractional order state-space systems, Journal of Vibrations and Control, Vol. 14(9/10), 1543-1556.

3. Dzieliński A., Sierociuk D., Sarwas G. (2009), Ultracapacitor parameters identification based on fractional order model, Proc ECC'09, Budapest.

4. Farina L., Rinaldi S. (2000), Positive Linear Systems; Theory and Applications, J. Wiley, New York.

5. Gantmacher F.R. (1959), The Theory of Matrices, Chelsea, New York.

6. Kaczorek T. (1992), Linear Control Systems, Research Studies Press and J.Wiley, New York.

7. Kaczorek T. (2001a), Externally and internally positive time-varying linear systems, Int. J. Appl. Math. Comput. Sci., Vol. 11(4), 957-964.

8. Kaczorek T. (2001b), Positive $1 D$ and 2D systems, Springer Verlag, London.

9. Kaczorek T. (2008a), Fractional positive continuous-time systems and their Reachability, Int. J. Appl. Math. Comput. Sci., Vol. 18(2), 223-228.

10. Kaczorek T. (2008b), Practical stability of positive fractional discretetime linear systems, Bull. Pol. Acad. Sci. Tech., Vol. 56(4), 313-317.

11. Kaczorek T. (2008c), Reachability and controllability to zero tests for standard and positive fractional discrete-time systems, Journal Européen des Systèmes Automatisés, JESA, Vol. 42(6-8), 769-787.

12. Kaczorek T. (2009), Asymptotic stability of positive fractional 2D linear systems, Bull. Pol. Acad. Sci. Tech., Vol. 57(3), 289-292.

13. Kaczorek T. (2011a), Checking of the positivity of descriptor linear systems by the use of the shuffle algorithm, Archive of Control Sciences, Vol. 21(3), 287-298.

14. Kaczorek T. (2011b), Controllability and observability of linear electrical circuits, Electrical Review, Vol. 87(9a), 248-254.

15. Kaczorek T. (2011c), Positive linear systems consisting of $n$ subsystems with different fractional orders, IEEE Trans. Circuits and Systems, Vol. 58(6), 1203-1210.

16. Kaczorek T. (2011d), Positivity and reachability of fractional electrical circuits, Acta Mechanica et Automatica, Vol. 5(2), 42-51. 
22. Klamka J. (1977), Minimum energy control of discrete systems with delays in control, International Journal of Control, Vol. 26(5), 737-744.

23. Klamka J. (1983), Minimum energy control of $2 \mathrm{D}$ systems in Hilbert spaces, System Sciences, Vol. 9(1-2), 33-42.

24. Klamka J. (1991), Controllability of Dynamical Systems, Kluwer Academic Press, Dordrecht.

25. Klamka J. (1993), Controllability of dynamical systems-a survey, Archives of Control Sciences, Vol. 2(3-4), 281-307.

26. Klamka J. (2010), Controllability and minimum energy control problem of fractional discrete-time systems, Chapter in "New Trends in Nanotechology and Fractional Calculus", Eds. Baleanu D., Guvenc Z.B., Tenreiro Machado J.A., Springer-Verlag, New York, 503-509.

Acknowledgment: This work was supported under work S/WE/1/11. 